\title{
Using Magnetic Resonance Myelography to Improve Interobserver Agreement in the Evaluation of Lumbar Spinal Canal Stenosis and Root Compression
}

\author{
Haider Najim Al-Tameemi ${ }^{1}$, Sattar Al-Essawi ${ }^{2}$, Mahmud Shukri ${ }^{3}$, Farah Kasim Naji ${ }^{4}$ \\ ${ }^{I}$ Department of Surgery/Radiology, College of Medicine, Kufa University, Najaf, Iraq \\ ${ }^{2}$ Department of Radiology, AL-Sader Medical City, Najaf, Iraq \\ ${ }^{3}$ Department of Surgery/Neurosurgery, College of Medicine, Kufa University, Najaf, Iraq \\ ${ }^{4}$ Department of Radiology, Marjan Teaching Hospital, Babylon, Iraq
}

Study Design: Cross-sectional retrospective study designed to assess interobserver agreement.

Purpose: To investigate if interobserver agreement using magnetic resonance imaging (MRI) in the evaluation of lumbar spinal canal stenosis and root compression can be improved upon combination with magnetic resonance myelography (MRM).

Overview of Literature: The interpretation of lumbar spinal MRl, which is the imaging modality of choice, often has a significant influence on the diagnosis and treatment of low back pain. However, using MRI alone, substantial interobserver variability has been reported in the evaluation of lumbar spinal canal stenosis and nerve root compression.

Methods: Hardcopies of 30 lumbar spinal MRI (containing a total of 150 disk levels) as well as MRM films were separately reviewed by two radiologists and a neurosurgeon. At each intervertebral disk, the observers were asked to evaluate the thecal sac for the presence and degree of spinal stenoses (mild, moderate, or severe) and presence of root canal compression. Interobserver agreement was measured using weighted kappa statistics.

Results: Regarding lumbar spinal canal stenosis, interobserver agreement between the two radiologists was moderate (kappa, 0.4) for MRI and good (kappa, 0.6) for combination with MRM. However, the agreement between the radiologist and neurosurgeon remained fair for MRI alone or in combination with MRM (kappa, 0.38 and 033, respectively). In the evaluation of nerve root compression, interobserver agreement between the radiologists improved from moderate (kappa, 0.57) for MRI to good (kappa, 0.73) after combination with MRM; moderate agreement between the radiologist and neurosurgeon was noted for both MRI alone and after combination with MRM (kappa, 0.58 and 0.56 , respectively).

Conclusions: Interobserver agreement in the evaluation of lumbar spinal canal stenosis and root compression between the radiologists improved when MRM was combined with MRI, relative to MRI alone.

Keywords: Lumbar region; Spinal stenosis; Nerve roots compression; Magnetic resonance imaging; Myelography

Received Jul 16, 2016; Revised Sep 2, 2016; Accepted Sep 22, 2016

Corresponding author: Haider Najim Al-Tameemi

Department of Surgery/Radiology, College of Medicine, Kufa University, Najaf 56001, Iraq

Tel: +964-33-340-952, Fax: +964-33-340-951, E-mail: haidernajim@yahoo.com 


\section{Introduction}

Low back pain (LBP) is a common clinical entity and considered as being one of most common causes of disability in younger individuals aged $\leq 45$ years [1]; consequently, it has a tremendous socioeconomic impact [2]. Among the many causes of LBP, acquired spinal stenosis caused by degenerative joint and disk diseases is responsible for the vast majority of cases [3]. Imaging evaluation of patients with spinal degenerative diseases often includes plain film radiography and magnetic resonance imaging (MRI), with the latter being the imaging modality of choice (Fig. $1 \mathrm{~A}, \mathrm{~B})$. Therefore, the interpretation of lumbar spinal MRI often has a significant role in LBP diagnosis and treatment $[4,5]$.

Magnetic resonance myelography (MRM) is a safe and noninvasive technique; compared with conventional myelography, it has approximately similar sensitivity in visualizing lumbar nerve roots [6], with the added advantages of no patient exposure to ionizing radiation or complications related to adverse reaction to intrathecal contrast material, such as arachnoiditis and adhesion [7]. The main principle of MRM, which is a heavily T2-weighted sequence, is that signals from non-water (solid) structures are reduced or nulled (black) so that the signal intensity from water (fluid) stands out clearly (bright) (Fig. 1C, D)
$[8,9]$.

Although several grading methods for the assessment of lumbar spinal canal stenosis have been suggested (including measuring the cross-sectional area), in practice, its severity is usually subjectively assessed. This is because no universal grading scale is currently used and no optimal method for imaging the spine has been established [10]. Consequently, substantial intra- and interobserver disagreements in the evaluation of lumbar spinal canal stenosis and root compression have been reported using MRI alone [11-13].

The objective of the present study was to investigate if MRM could be used to improve the interobserver agreement between a radiologist and non-radiologist (neurosurgeon) in the evaluation of lumbar spinal canal stenosis and nerve root compression using MRI.

\section{Materials and Methods}

The study was approved by the regional Institutional Ethical Committee.

A cross-sectional analytic retrospective study was conducted; the study comprised 30 patients (18 females and 12 males, aged 25-65 years) at the MRI unit of the Middle Euphtare Neuroscience Center, Al-Sader Medical City, AlNajaf, Iraq over a 6-month period. The study sample was
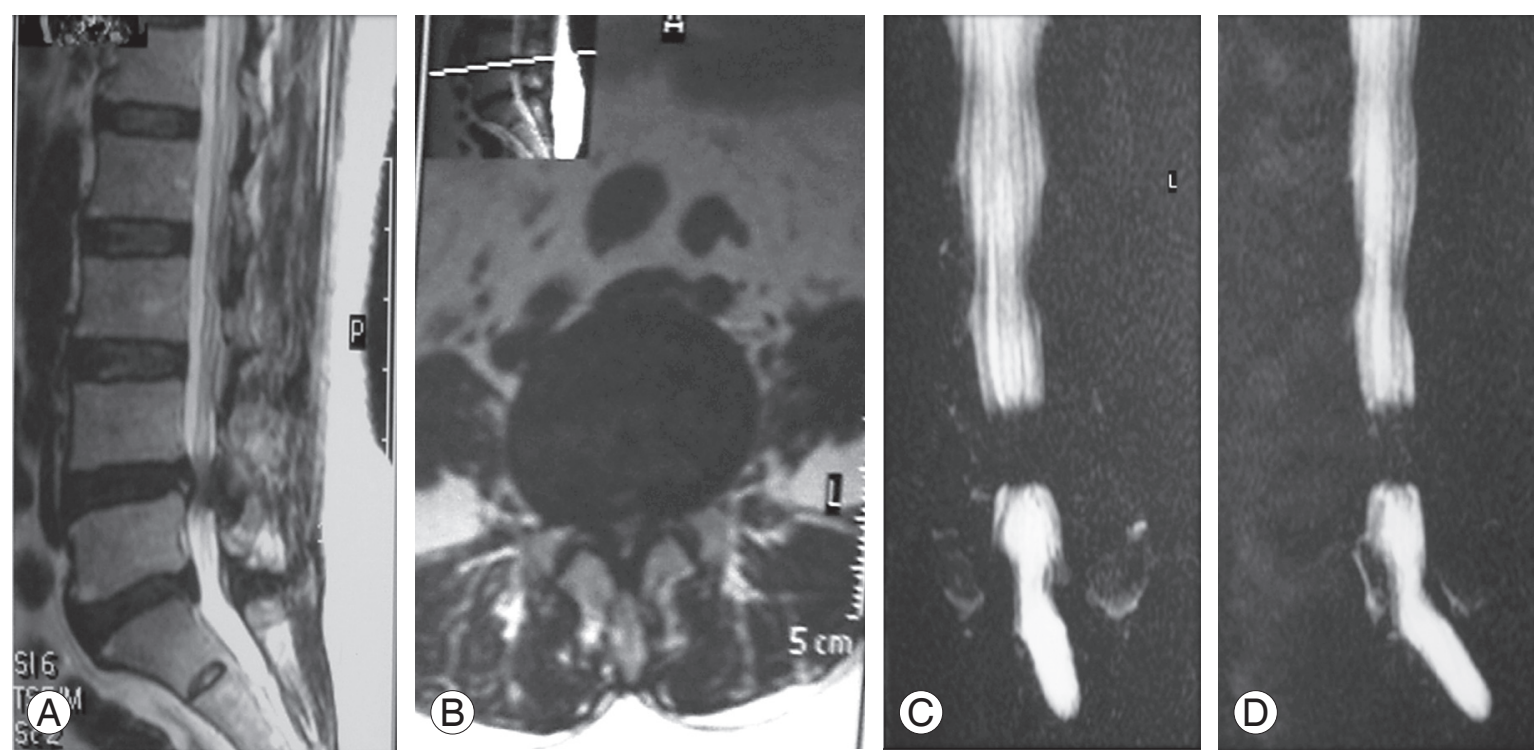

Fig. 1. A 65-year-old woman with low back pain. Sagittal (A) and axial (B) T2-weighted magnetic resonance turbo spin echo images (repetition time $=2,363 \mathrm{msec} / \mathrm{echo}$ time=100 $\mathrm{msec}$ ) showing degeneration of the 4 th-5th lumbar intervertebral disk with posterior bulge together with ligamentum flavum hypertrophy, causing severe lumbar spinal canal stenosis and bilateral root compression. Coronal (C) and sagittal (D) magnetic resonance myelography images (repetition time $=8,000 \mathrm{msec} / \mathrm{echo}$ time $=1,000 \mathrm{msec}$ ) confirming the presence of severe lumbar spinal canal stenosis at the 4th-5th disk level. 
randomly selected from patients who had already been referred to the MRI unit for lumbosacral evaluation, with the indication being either LBP, radiculopathy, or both. Cases with variable degrees of lumbar spinal canal stenoses and root compression were included. MRI examinations with incomplete sequences or artifacts and cases with lumbosacral mass or trauma were excluded.

\section{MRI and MRM examinations}

All cases were examined using the same MRI device (Philips Achieva 1.5 Tesla, Netherland, 2010). During lumbosacral evaluation, MRI and MRM were performed using the following imaging parameters:

(1) Sagittal T1-weighted images: turbo spin echo (TSE) echo time $(\mathrm{TE})=8 \mathrm{msec}$ and repetition time $(\mathrm{TR})=500$ msec.

(2) Sagittal T2-weighted images: TSE TE $=100 \mathrm{msec}$ and $\mathrm{TR}=4,000 \mathrm{msec}$.

(3) Axial T2-weighted images: $\mathrm{TE}=120 \mathrm{msec}$ and $\mathrm{TR}=4,000 \mathrm{msec}$.

(4) MRM (heavily T2-weighted images): TE $=1,000$ msec and TR=8,000 msec.

\section{Image analysis}

Hardcopies of 30 conventional lumbosacral MRI films with a total of 150 disk levels, as well as hardcopies of their corresponding MRM films, were separately reviewed by two blinded board-certified radiologists and one boardcertified neurosurgeon. At first, each observer evaluated the MRI films alone. Then, the observer re-evaluated each case, but this time in adjunct with the MRM film. Using structured formula sheets, the observers were asked to assess specific parameters at each level of the 150 disks, including (1) nerve root compression (present or absent); (2) severity of lumbar spinal canal stenosis using the 4-grade classification of severity reported by Lee et al. [14], which is based on the degree of separation of the cauda equina on T2-weighted axial images, as follows: grade 0 , no lumbar spinal canal stenosis without obliteration of the anterior cerebrospinal fluid space; grade 1, mild stenosis with separation of all cauda equina; grade 2 , moderate stenosis with some cauda equina aggregated, making it impossible to visually separate them; and grade 3 , severe stenosis with none of the cauda equina separated.

\section{Statistical analysis}

Data were statistically analyzed using SPSS ver. 2.1 (SPSS Inc., Chicago, IL, USA). Interobserver reliability was assessed using weighted kappa statistics to determine con-

Table 1. Demographic data of the 30 patients included in the study

\begin{tabular}{|c|c|c|}
\hline Parameter & MRI & MRM \\
\hline Age (yr) & $50.7 \pm 11.17$ & \\
\hline \multicolumn{3}{|l|}{ Sex } \\
\hline Male & 12 & \\
\hline Female & 18 & \\
\hline Weight (kg) & $89.3 \pm 10.7$ & \\
\hline \multicolumn{3}{|c|}{$\begin{array}{l}\text { Grade of lumbosacral canal } \\
\text { stenosis ( } 150 \text { levels })^{\text {a) }}\end{array}$} \\
\hline \multicolumn{3}{|l|}{ R1 } \\
\hline Grade 0 & 107 & 104 \\
\hline Grade 1 & 16 & 22 \\
\hline Grade 2 & 17 & 10 \\
\hline Grade 3 & 10 & 14 \\
\hline \multicolumn{3}{|l|}{$\mathrm{R} 2$} \\
\hline Grade 0 & 76 & 87 \\
\hline Grade 1 & 41 & 37 \\
\hline Grade 2 & 10 & 9 \\
\hline Grade 3 & 23 & 17 \\
\hline \multicolumn{3}{|l|}{$\mathrm{N}$} \\
\hline Grade 0 & 109 & 115 \\
\hline Grade 1 & 22 & 18 \\
\hline Grade 2 & 9 & 5 \\
\hline Grade 3 & 10 & 12 \\
\hline \multicolumn{3}{|c|}{ Roots compression on MRI } \\
\hline \multicolumn{3}{|l|}{ R1 } \\
\hline Yes & 66 & \\
\hline No & 84 & \\
\hline \multicolumn{3}{|l|}{$\mathrm{R} 2$} \\
\hline Yes & 55 & \\
\hline No & 95 & \\
\hline \multicolumn{3}{|l|}{$\mathrm{N}$} \\
\hline Yes & 34 & \\
\hline No & 116 & \\
\hline
\end{tabular}

Values are presented as mean \pm standard deviation $\mathrm{MRI}$, magnetic resonance imaging; MRM, magnetic resonance myelogram; R1, first radiologist; $R 2$, second radiologist; N, neurosurgeon.

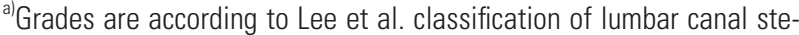
nosis [14] 
sistency and agreement among the observers. The interpretation of kappa measurements was in accordance with Altman's practical statistics [15]: poor agreement, $<0.20$; fair agreement, 0.20-0.39; moderate agreement, $0.40-0.59$; good agreement, 0.60-0.79; and very good agreement, 0.80-1.00.

No patient consent was obtained because of the noninterventional nature of the study, with the confidentiality of patient's personal data being preserved.

\section{Results}

The 30 patients included in this study had a mean age of 50.7 years (standard deviation, \pm 11.17 years) (Table 1 ). After the evaluation of the 150 intervertebral levels in all 30 MRI lumbosacral examinations using MRI and then MRM, interobserver agreement before and after using MRM was compared. The following results were obtained:

\section{Lumbosacral spinal canal stenosis}

The overall agreement between the two radiologists regarding the severity of lumbar spinal canal stenosis was better for MRM (good) than for MRI (moderate), with kappa values of 0.6 and 0.4 , respectively (Table 2 ). On the other hand, there was no significant change in interobserver agreement (fair) between the radiologist and neurosurgeon; the kappa values were 0.38 and 0.33 , respectively (Table 2).

\section{Nerve root compression}

The agreement between the two radiologists improved from moderate (kappa value, 0.57 ) when using MRI to good (kappa value, 0.7) when using MRM (Table 3). However, between the radiologist and the neurosurgeon, there was no significant change in agreement in the evaluation of root compression using MRI and MRM (moderate for each, with kappa values of 0.58 and 0.56 , respectively) (Table 3).

\section{Discussion}

Although MRI is the modality of choice for assessing disk abnormalities [16], MRM can be added to routine lumbar spinal MRI scans and may be used to help confirm abnormalities noted via conventional MRI in selected cases [17]. In clinical practice, the role of MRM in improving the interpretation of lumbosacral MRI can be inferred by comparing interobserver agreement between two different radiologists and between a radiologist and non-radiologist (neurosurgeon) on MRI and then on MRM. Using this approach, lumbar spinal canal stenosis and nerve root compression were assessed in the current study.

In lumbar spinal canal stenosis, the reported variability in intraobserver and interobserver reliability is in general more pronounced when qualitative rather than quantitative measures are applied $[12,13]$, and interobserver agreement is particularly low between clinicians of different specialties (e.g., radiologists vs. surgeons) $[18,19]$.

In our study, MRM clearly improved the agreement between radiologists (Table 2); however, MRM had no significant effect on the agreement between the radiologist and neurosurgeon. This implies that MRM tends to result in a more objective assessment between radiologists than between a radiologist and non-radiologist. This improve-

Table 2. Comparison between interobserver agreement using MRI and MRM in the evaluation of spinal stenosis

\begin{tabular}{lllll} 
Parameter & Observers & MRI (kappa) & MRM (kappa) & Change \\
Spinal stenosis & R1 and R2 & Moderate (0.4) & Good (0.6) & Improved \\
Spinal stenosis & R2 and N & Fair (0.38) & Fair (0.33) & No significant improvement \\
\hline
\end{tabular}

MRI, magnetic resonance imaging; MRM, magnetic resonance myelogram; R1, first radiologist; R2, second radiologist; $N$, neurosurgeon.

Table 3. Comparison between interobserver agreement using MRI and MRM in the evaluation of nerve root compression

\begin{tabular}{lllll} 
Parameter & Observers & MRI (kappa) & MRM (kappa) & Change \\
Root compression & R1 and R2 & Moderate (0.57) & Good (0.7) & Improved \\
Root compression & R2 and N & Moderate (0.58) & Moderate (0.56) & No significant improvement \\
\hline
\end{tabular}

MRI, magnetic resonance imaging; MRM, magnetic resonance myelogram; R1, first radiologist; R2, second radiologist; N, neurosurgeon. 
ment in agreement when using MRM was consistent with the results of a study by Song et al. [20] involving the evaluation of 100 patients with multilevel disease, where the interobserver reliability of MRI in identifying and grading lumbar spinal canal stenosis was improved with the use of MRM.

Similarly, regarding the presence or absence of root compression, we found that MRM increased the agreement from moderate to good between the two radiologists, whereas no improvement was observed between the radiologist and neurosurgeon. Hence, the addition of MRM also resulted in a more objective reading of MRI examinations, but only between the radiologists.

Because of the large interobserver variability in diagnosing root compression and lumbar spinal canal stenosis using MRI, it is recommended that MRI findings alone should not be used when assessment is required for a surgical decision; in these cases, a higher-Tesla MRI would be a better alternative [21]. A score developed by Azimi et al. [22] is also generally considered reliable and valid, and it can be used in the decision-making process with respect to surgical intervention. Because of the improved interobserver agreement, at least between radiologists, we believe that adding MRM could be another solution to help surgeons make surgical decisions more judiciously. However, this solution will be more clinically applicable when interdepartmental agreement is also good, which was not attainable in this study. The interdepartmental disagreement seen in this study may not exactly reflect the actual practice and cannot be generalized because only one non-radiologist (neurosurgeon) was involved in the assessment. However, we believe that this difference cannot be ignored and may be explained by several factors, both individual and departmental, including but not limited to different knowledge, skills, and clinical experiences with MRI and MRM as well as varied educational backgrounds.

There were some limitations to this study. First, we used only a single classification system for lumbosacral canal stenosis grading without utilizing direct measurement of spinal canal dimensions. However, while the latter would be more objective and reproducible, we believe that in the daily practice, it is less commonly used than subjective assessment methods (including the one we chose). Second, we evaluated hardcopies and not digital images on the workstation. This is because, unfortunately, we have not yet established a picture archiving and communica- tion system, and data transfer is still largely dependent on printed films. Finally, only one nonradiologist was involved in this study, so interdepartmental variability cannot be definitely accepted due to a small number of observers. Nevertheless, we believe that this study has shed some light on this variability, and further studies need to be conducted to confirm our observations.

\section{Conclusions}

MRM can improve the interpretation of lumbosacral MRI examinations because of the higher level of agreement achieved than when using MRI alone. MRM can result in better and more reproducible data transfer, particularly between radiologists.

Measures to reduce interobserver disagreement between radiologists themselves and between radiologists and non-radiologists need to be acted upon and might include but not be limited to the following: (1) a scientific conference with radiologist and non-radiologist participation to reach a reliable consensus regarding the evaluation of lumbosacral MRI interpretation and (2) creation of a standardized grading system of lumbosacral spinal stenosis to maintain a reproducible and less operatordependent interpretation.

In addition, further quality control studies, encompassing more specific radiological parameters in the evaluation of lumbosacral spinal MRI, should be conducted.

\section{Conflict of Interest}

No potential conflict of interest relevant to this article was reported.

\section{References}

1. Takahashi Y, Ohtori S, Takahashi K. Peripheral nerve pathways of afferent fibers innervating the lumbar spine in rats. J Pain 2009;10:416-25.

2. Modic MT, Ross JS. Lumbar degenerative disk disease. Radiology 2007;245:43-61.

3. Moreland LW, Lopez-Mendez A, Alarcon GS. Spinal stenosis: a comprehensive review of the literature. Semin Arthritis Rheum 1989;19:127-49.

4. Deyo RA, Mirza SK, Turner JA, Martin BI. Overtreating chronic back pain: time to back off? J Am Board Fam Med 2009;22:62-8. 
5. Wang C, Auerbach JD, Witschey WR, Balderston RA, Reddy R, Borthakur A. Advances in magnetic resonance imaging for the assessment of degenerative disc disease of the lumbar spine. Semin Spine Surg 2007;19:65-71.

6. Pui MH, Husen YA. Value of magnetic resonance myelography in the diagnosis of disc herniation and spinal stenosis. Australas Radiol 2000;44:281-4.

7. Figueroa RE, Stone JA. MR imaging of degenerative spine disease: MR myelography and imaging of the posterior spinal elements. In: Castillo $\mathrm{M}$, editor. Spinal imaging, state of art. Philadelphia: Hanley \& Belfus; 2001. p.105-22.

8. Ferrer P, Marti-Bonmati L, Molla E, Casillas C. Value of MR myelography in the diagnosis of the spine disorders. Med Clin (Barc) 2000;115:366-9.

9. O'Connell MJ, Ryan M, Powell T, Eustace S. The value of routine MR myelography at MRI of the lumbar spine. Acta Radiol 2003;44:665-72.

10. Jacob DS. Degenerative diseases of the spine. In: Hagga JR, editors. CT and MRI of the whole body. 5th ed. Philadelphia: Mosby/Elsevier; 2009. p.756-62.

11. Kovacs FM, Martinez C, Arana E, et al. Uncertainties in the measurement of lumbar spinal stenosis at MR imaging: are they clinically relevant? Radiology 2012; 263:310-1.

12. Lurie JD, Doman DM, Spratt KF, Tosteson AN, Weinstein JN. Magnetic resonance imaging interpretation in patients with symptomatic lumbar spine disc herniations: comparison of clinician and radiologist readings. Spine (Phila Pa 1976) 2009;34:7015 .

13. Arana E, Royuela A, Kovacs FM, et al. Lumbar spine: agreement in the interpretation of 1.5-T MR images by using the Nordic Modic Consensus Group classification form. Radiology 2010;254:809-17.

14. Lee GY, Lee JW, Choi HS, Oh KJ, Kang HS. A new grading system of lumbar central canal stenosis on MRI: an easy and reliable method. Skeletal Radiol 2011;40:1033-9.

15. Altman DG. Practical statistics for medical research. London: Chapman and Hall; 1991.

16. Plecha DM. Imaging of bone marrow disease in the spine. Semin Musculoskelet Radiol 2000;4:321-7.

17. Thornton MJ, Lee MJ, Pender S, McGrath FP, Brennan RP, Varghese JC. Evaluation of the role of magnetic resonance myelography in lumbar spine imaging. Eur Radiol 1999;9:924-9.

18. Carrino JA, Lurie JD, Tosteson AN, et al. Lumbar spine: reliability of MR imaging findings. Radiology 2009;250:161-70.

19. Lurie JD, Tosteson AN, Tosteson TD, et al. Reliability of readings of magnetic resonance imaging features of lumbar spinal stenosis. Spine (Phila Pa 1976) 2008; 33:1605-10.

20. Song KS, Jang EC, Jung HJ, Kim KW, Yu H. Observer variability in the evaluation of multiple lumbar stenosis by routine MR: myelography and MRI. J Spinal Disord Tech 2008;21:569-74.

21. Drew R, Bhandari M, Kulkarni AV, Louw D, Reddy K, Dunlop B. Reliability in grading the severity of lumbar spinal stenosis. J Spinal Disord 2000;13:253-8.

22. Azimi P, Mohammadi HR, Benzel EC, Shahzadi S, Azhari S. Lumbar spinal canal stenosis classification criteria: a new tool. Asian Spine J 2015;9:399-406. 